REVIEW ARTICLE

\title{
Overview of Safety Assessment and Toxicological Screening of Dermal Formulations
}

\author{
Subramani Parasuraman ${ }^{1}$, Subramani Balamurugan ${ }^{2}$, Raipan Vanishya $^{3}$
}

\begin{abstract}
The skin is one of our largest organs and the delivery of drugs via the skin is gorgeous and also challenging vicinity in research. The dermal formulations are used for cosmetic and therapeutic purposes by various age groups. Hence, the safety data are essential for these preparations and required long-term toxicity testing is essential to prevent delayed effects on users/consumers. In recent years, use of dermal preparations including cosmetic products has ever increased globally which exhibiting potential health risks including mild hypersensitivity and lethal intoxication. Hence, testing any formulation including dermal preparations for its toxicity is the basic requirement in most toxicological frameworks. Moreover, many of the dermal preparations are available over-the-counter. Hence, the data on the safety of these preparations are very essential, which can be studied using preclinical models, preferably using cell lines or animals. The purpose of the current review is to summarize the toxicity testing methods for dermal preparations.
\end{abstract}

Keywords: Draize's criteria, Globally harmonized system, Organisation for Economic Co-operation and Development, Toxicity.

SBV Journal of Basic, Clinical and Applied Health Science (2020): 10.5005/jp-journals-10082-02258

\section{INTRODUCTION}

The skin is one of our largest organs with an average surface of about $2 \mathrm{~m}^{2}$. Functions of the skin include protecting the body against exogenous and xenobiotic impacts, multisensory, hormonal, and thermoregulation. ${ }^{1}$ Delivery of drugs via the skin is gorgeous and also challenging vicinity in research. The advancement of the research is resulting in the development of a larger number of transdermal drug delivery system for hydrophilic drugs, macromolecules, and conventional hydrophobic smallmolecule drugs. ${ }^{2}$

Transdermal drug delivery system is a discrete dosage forms under the class of controlled drug delivery which use the skin as the main route of drug delivery for various application. ${ }^{3}$ Bath additive, collodion, cream, cutaneous preparations (including stick, emulsion, foam, liquid, paste, powder, solution, spray, suspension), gel, impregnated dressing, medicated nail lacquer, ointment, poultice, shampoo, solution for iontophoresis, transdermal patch, medicated plaster, plaster for provocation test, powder for the dip solution, solution for the skin-prick test, solution for the skin-scratch test, transdermal system, and cutaneous patch are the cutaneous and transdermal preparations. ${ }^{4}$ The dermal preparations are used for cosmetic and therapeutic purposes. Transdermal drug delivery systems are mostly inexpensive and these formulations are designed to deliver drugs from 1-7 days. ${ }^{5}$ The global market value of dermal or skincare products is $3,471.5$ million USD in 2018 and it may increase to approximately 6,303 million USD by $2026 .{ }^{6}$ The rising number of skin disorders and geriatric population globally may increase the needs of dermal fillers in the future. ${ }^{7}$

In recent decades, the use of dermal preparations including cosmetic products has ever increased globally which exhibiting potential health risks including mild hypersensitivity and lethal intoxication. ${ }^{8,9}$ In skincare formations, toxic substances, such as 1,4-dioxane, benzalkonium chloride, butylated hydroxytoluene, diazolidinyl urea, formaldehyde, imidazolidinyl urea, isothiazolinone derivatives, methyldibromo glutaronitrile, parabens derivatives,

\footnotetext{
1,3 Department of Pharmacology, Faculty of Pharmacy, AIMST University, Bedong, Kedah, Malaysia

${ }^{2}$ Directorate of Drugs Control Administration, Chennai, India

Corresponding Author: Subramani Parasuraman, Department of Pharmacology, Faculty of Pharmacy, AIMST University, Bedong, Kedah, Malaysia, Phone: +60 108826480, e-mail: parasuphd@gmail.com

How to cite this article: Parasuraman S, Balamurugan S, Vanishya R. Overview of Safety Assessment and Toxicological Screening of Dermal Formulations. J Basic Clin Appl Health Sci 2020;3(3):96-103.

Source of support: Nil

Conflict of interest: None
}

paraformaldehyde, phenoxyethanol, phthalates, polyethylene glycols, sodium laureth sulfate, and trace heavy metals, have used ingredients that can cause health risk to the human including cytotoxicity, genotoxicity, mutagenicity, neurotoxicity, and estrogenicity. ${ }^{8}$ Hence, testing of any formulations including dermal preparations for their toxicity is the basic requirements in most toxicological frameworks.

The toxicological study begins with Paracelsus, who investigated the specific chemicals which are responsible for animal and plant toxicity. But, toxicity testing is gained much attention in the early 1960 s, because of the thalidomide tragedy. The guidelines for toxicity testing of pharmaceutical compounds/substances were outlined by the International Conference on Harmonization (ICH) and the Organisation for Economic Co-operation and Development (OECD) in the late $1980 \mathrm{~s} .{ }^{10}$ Compare to other formulations/pharmaceutical dosage forms, the dermal formulations/preparations are used for cosmetic and treatment purposes by neonates to geriatric people. Moreover, many of the dermal preparations are available over-thecounter. Hence, the data on the safety of these preparations are very essential, which can be studied using preclinical models, preferably using cell lines or animals. The purpose of the current review is to summarize the toxicity testing methods for dermal preparations. 


\section{Toxicity Testing Methods}

- Animal models:

- Acute dermal toxicity

- Subchronic dermal toxicity

- Dermal irritation

- Skin corrosion test

- Skin sensitization test

- Phototoxicity

- Non-animal models

- In vitro skin irritation assay

- In vitro skin corrosion test

- In vitro skin sensitization test

- Phototoxicity and photoallergy

- Skin genotoxicity.

\section{Acute Dermal Toxicity}

As per Occupational Safety and Health Administration (OSHA), acute dermal toxicity by the dermal route refers "to those adverse effects occurring following a single dermal (skin) exposure to a substance, or multiple such exposures within 24 hours". ${ }^{11}$ The dose is known as the quantity of test compound used and is expressed as a test compound weight per unit weight of test animal. The $\mathrm{LD}_{50}$ (median lethal dose) is a single dose statistically derived from a substance that, when administered/applied to the skin, can be likely to cause death in $50 \%$ of treated animals. The $\mathrm{LD}_{50}$ value is expressed in terms of test product weight per unit weight of test animal $(\mathrm{mg} / \mathrm{kg})^{12}$

As per recommendations, OECD/Organisation de coopération et de développement économiques (OCDE) adopted 2017 test guidelines (TG) 402, acute dermal toxicity test is performed in single sex (female gender is preferable) rats (when the test is conducted in male animals, adequate justification must be provided), are administered with test compound in a stepwise procedure using the suitable fixed doses. The study is started with a low dose/safe dose at the concentration predictable to produce clear toxic signs without causing severe toxic effects or mortality. Additionally, the test compound may be tested at lower or higher fixed doses on groups of animals, depending on the absence or presence of signs of toxicity. This procedure is continued until when no effects are observed at the highest dosage or the dose causing toxicity or no more than one death, or when deaths occur at the lowest dose. ${ }^{13}$

Young, adult, nulliparous, and non-pregnant female rats with the age of $8-10$ weeks old and body weight (BW) of $200-300 \mathrm{~g}$ (interindividual variation of BW should be $\pm 20 \%$ ) are used for acute dermal toxicity testing. One day, prior to the experiment, all fur/hair is removed from the dorsal/flank region of the test animals (i.e., at least $10 \%$ of the total body surface area). To reduce handling stress to the animals, aesthetics can be used.

On the day of the experiment, the test compound (if the test compound is solid, the compound is moistened sufficiently with a suitable vehicle) is applied as uniformly over the exposed region of dorsal/flank skin. After the application of the test compound, the exposed area can be closed with a gauze dressing and non-irritating tape during a 24-hour exposure period. During the 24-hour exposure period, animals can be housed individually in rodent cages. After exposure period of 24 hours, residual test compound is removed using an appropriate solvent or water. ${ }^{13}$

In acute dermal toxicity studies, the test compound is administered to single animals in a sequential manner. In the main study, two animals are used for any selected dose level. The test compound is studied at the different dose levels up to $2,000 \mathrm{mg} / \mathrm{kg}$ based on the Globally Harmonized System (GHS) categories for acute dermal toxicity. Under GHS and Bazard classification, chemical are divided into five acute toxicity (dermal) categories using the $\mathrm{LD}_{50}$ values viz. category 1 ( $\leq 50 \mathrm{mg} / \mathrm{kg} \mathrm{BW}$ ), category 2 (>50 and $\leq 200 \mathrm{mg} / \mathrm{kg} \mathrm{BW}$ ), category 3 (>200 and $\leq 1000 \mathrm{mg} / \mathrm{kg} \mathrm{BW}$ ), category 4 (>1000 and $\leq 2000 \mathrm{mg} / \mathrm{kg} \mathrm{BW}$ ), and category 5 (>2000). Acute toxicity category 1 represents the most severe toxicity. ${ }^{14}$

Animals are observed at least once during the first 0.5 hour and periodically for first 24 hours and daily for next 14 days. During the experiment, the animals are monitored for changes in skin and hair, mucous membranes, eyes and also nervous systems, circulatory, respiratory, and somatomotor activity. In addition, after removal of the test compound, the testing site is observed at 24,48 , and 72 hours using the Draize criteria (observed for signs of erythema and edema) and this study results may use to get a waiver for a separate in vivo skin irritation study. ${ }^{13}$

If no skin irritation is observed in dermal acute toxicity studies, the use of animals for dermal irritancy testing can be avoided. ${ }^{15}$

\section{Subchronic Dermal Toxicity}

As per recommendations, OECD/OCDE adopted 1981TG 411, subchronic dermal toxicity is performed. ${ }^{16}$

In subchronic dermal toxicity testing, the test compound is applied daily (one dose/group) to the animal skin for a period of 90 days. During the experiment, the animals are monitored for any toxic signs. ${ }^{17}$

The adult rat, guinea pig, or rabbit are used as experimental animals. Interindividual variation of BW should be $\pm 20 \%$. Other animal species can be used for the investigation but their use should be justified. At least 20 healthy animals ( 10 males and 10 females) are used at each dose level of test compound and female animals are non-pregnant and nulliparous. The number of animals in each group can be increased, if the experimental protocol requires interim sacrifices. Apart from control and investigational groups, a satellite group of 20 animals ( 10 males and 10 females) may be administered with the high-dose level of test compound for 90 days and observed for persistence, reversibility, or delayed incidence of toxic effects for 28 days posttreatment. ${ }^{16}$

One day, prior to the experiment, all fur/hair is removed from the dorsal/flank region of the test animals (i.e., at least $10 \%$ of the total body surface area). To reduce handling stress to the animals, esthetics can be used. During the experiment, the test compound should (if the test compound is solid, the compound is moistened sufficiently with a suitable vehicle) be applied as uniformly over the exposed region of dorsal/flank skin. After the application of the test compound, the exposed area can be closed with a gauze dressing and non-irritating tape. Also, ensure that the animals are not ingesting the test compound. During the study period, the animals are monitored daily and signs of toxicity are noted, including the time of onset, their degree, and duration. The animals are observed for changes in skin and hair, mucous membranes, eyes and also nervous systems, circulatory, respiratory, and somatomotor activity. During the experiment, if any animal dies, it should be necropsied and biological samples are collected, subjected to biochemical and histological analyzes. At the end of the study period, all survivors in all experimental groups except satellite treatment groups are necropsied and possible biological samples are collected for laboratory (biochemical and hematological) investigation. ${ }^{16,18}$

At least three dose levels of test compound are tested with a control or vehicle control. The exposure period is at least 6 hours/ 
day and the application of the test compound is made at similar times each day, and the amount of test compound applied adjusted at intervals (weekly/biweekly) based on animal BW. ${ }^{17}$ In subchronic dermal toxicity testing, any adverse effect occurs; the study is repeated with the daily dermal application of test compound to the experimental animals for part of an animal life span (not exceeding $10 \%$ of animal total life span). ${ }^{16}$

Caution: If the application of the test compound produces severe skin irritation or any allergic reactions, the concentrations of the test compound are reduced. If the skin has been severely injured, the study can be terminated and the new study is planned with lower concentrations of the test compound.

\section{Dermal (Skin) Irritation Test}

Skin is frequently exposed to cosmetic products and it should not cause skin irritation and skin corrosion. Hence, these products is tested for skin irritation and skin corrosion as part of the overall safety assessment process. ${ }^{19}$ Dermal irritation is production of reversible injury/damage of the skin after the application of a test compound for up to 4 hours. ${ }^{20}$ In this test, the test compound is applied in a single dose to the animals' skin and degree of irritation are assessed at specified intervals.

Young adult albino rabbits are used as an animal model for dermal irritation test. Other animal species can be used for the investigation but their use should be justified. A day before the administration of the test compound, hair is removed on the dorsal surface of the trunk of the animals, and adequate care is taken to prevent abrading the skin. During the experiment, the animals are housed individually.

On the day of the experiment, test compound (if the test compound is solid, the compound is moistened sufficiently with a suitable vehicle) is applied to a small area (approximately $6 \mathrm{~cm}^{2}$ ) of skin and closed with a gauze dressing and non-irritating tape. The animal is applied sequentially with up to three test patches. After 3 minutes, the first patch is removed and observed for any skin reactions. If no significant or serious skin injury/reactions are observed, the second patch is applied at a different location and removed after 1 hour and observed for any skin reactions. If no serious skin reaction is observed, the third patch is applied at a different location and removed after 4 hours and the response is graded.

After three sequential exposures, any corrosive effect is observed, the test can be terminated immediately. If, no corrosive effect is observed after removal of the last patch, the animal is observed at specific times for irritant responses for 14 days.

The test compound is not likely to produce corrosion during the observation period, but can be irritating; the study can be performed with a single patch to one animal for 4 hours. If the corrosive effect is not observed, the experiment can be repeated with another two animals.

Observation: The test sites are scored for erythema and edema at $1,24,48$, and 72 hours postexposure based on the GHS categories for acute toxicity hazard categories. ${ }^{21}$

\section{Skin Corrosion Test}

Skin corrosion is the production of irreversible skin damage that has been showed as visible necrosis through the epidermis and the dermis following the application of the test compound. ${ }^{22}$

The test protocol and animal models are similar to the dermal (skin) irritation test. The test is carried out as per the method described in OECD TG 404. Corrosive reactions are characterized by bleeding, bloody scabs, ulcers, discoloration, complete areas of alopecia, and scars. In corrosion test, animals are observed for irreversible skin damage including visible necrosis, following the application of a test compound for up to 4 hours. The histopathological study is performed at the end of the study to evaluate questionable lesions. ${ }^{20}$

\section{Skin Sensitization Test}

Skin sensitizers are chemical substances that cause an allergy after repeated contact with the skin. ${ }^{23}$ Skin sensitization is assessed using the Draize test, guinea pig maximization test (GPMT), Buehler test, and murine local lymph node assay (LLNA). ${ }^{10}$

\section{Guinea Pig Maximization Test}

Guinea pigs are used as an animal model for GPMT. A minimum of 10 and 5 animals is used in the treatment and control group, respectively. The use of 20 and 10 animals in the treatment and control group, respectively, is strongly recommended. In the flank region, hair is removed by shaving or by chemical depilation, and adequate care is provided to prevent skin abrasion. Protocol for GPMT is summarized in Table $1 .{ }^{24}$

\section{Buehler Test}

Guinea pigs are used as an animal model for the Buehler test. A minimum of 20 and 10 animals is used in the treatment and control group, respectively. On the dorsal surface, hair is removed by shaving or by chemical depilation, and adequate care is provided to avoid abrading the skin. Protocol for the Buehler test is summarized in Table $2 .^{24}$

\section{Murine LLNA}

Local lymph node assay has largely superseded the GPMT and the Buehler test. Mice are used as experimental model for LLNA. Animals are observed for skin lesions prior to the experiment. If any animals have skin lesions, that should be excluded from the experiment. The animals are divided into five groups viz., negative/ vehicle control group, positive control, and three concentrations of the test compound with minimum four animals per group. The LLNA procedure is as follows:

Day 1: Weight of the mouse is recorded and test compound is applied ( $25 \mu \mathrm{L} /$ ear). A $25 \mu \mathrm{L} /$ ear of the test compound/positive control or vehicle is applied to the dorsum of both ears of each mouse in respective groups.

Day 2 and 3: Repeat the application technique as carried out on day 1 . The animals are monitored for the clinical signs of toxicity or local irritation after drug application. The animals also observed for erythema and scored (score 0: no visual effect; score 1: slight erythema; score 2: well-defined erythema; score 3: moderate to severe erythema; and score 4: eschar).

Day 4 and 5: No treatment. Ear thickness is measured on day 1, day 3 , and day 6 using a thickness gauge.

Day 6: The weight of the mice is recorded. Each mouse is injected with $250 \mu \mathrm{L}$ of sterile phosphate-buffered saline (PBS) containing 2 $\mu \mathrm{Ci}$ of ${ }^{125} \mathrm{I}$-iododeoxyuridine $\left({ }^{125} \mathrm{IU}\right)$ or $20 \mu \mathrm{Ci}$ of tritiated $\left({ }^{3} \mathrm{H}\right)$-methyl thymidine and $10^{-5} \mathrm{M}$ fluorodeoxyuridine through the lateral tail vein. After 5 hours, the mouse is euthanized and the draining lymph nodes are collected from both ears and placed in PBS. A single-cell suspension of lymph node cells (LNCs) is prepared for each mouse. LNC is washed with a PBS and the deoxyribonucleic acid (DNA) precipitated with $5 \%$ trichloroacetic acid (TCA) at $4^{\circ} \mathrm{C}$ for approximately 18 hours. 
For the ${ }^{125} \mathrm{IU}$ method, the $1 \mathrm{~mL}$ TCA pellet is transferred into gamma-counting tubes and incorporation of ${ }^{125} \mathrm{IU}$ is determined by gamma counting and expressed as disintegrations per minute $(\mathrm{dpm}) /$ mouse. For the ${ }^{3} \mathrm{H}$-methyl thymidine method, pellets are resuspended in $1 \mathrm{~mL}$ TCA and transferred to $10 \mathrm{~mL}$ of scintillation fluid and incorporation of ${ }^{3} \mathrm{H}$-methyl thymidine is measured by $\beta$-scintillation counting and expressed as $\mathrm{dpm} /$ mouse. ${ }^{25}$

\section{Phototoxicity}

Terrestrial animals which include humans are continuously exposed to sunlight irradiation. The skin, an organ with largest surface area in the body, is normally affected by sunlight. Indeed, skin-associated problems, such as sunlight-induced skin burn, photoaging, rash, hyperpigmentation, and skin cancers, are generally observed nowadays. ${ }^{26}$ Incidentally, the skin is often exposed to solar irradiation and exogenous xenobiotics which may produce phototoxicity. Photoreactivity is a chemical reaction exemplified by photosynthesis and photocleavage. In the end, the reactions from solar irradiation cause cytotoxicity to skin cells, and eventually, inflammation of the skin. ${ }^{26,27}$

Healthy, adult, male, albino Hartley strain guinea pigs (500 \pm $100 \mathrm{~g} \mathrm{BW}$ ) are used. The test compound is administered orally or topically. Two hours after the oral dose or 15 minutes after the topical application (for topical administration, the hair is removed from the site of drug administration), the guinea pigs are immobilized on a wooden board and irradiated with long-wave ultraviolet (UV) at $3 \mathrm{~J} / \mathrm{cm}^{2}$. The phototoxic ability is depending on the time interval between drug administration and UV irradiation. ${ }^{28}$ After 24,48 , and 72 hours of irradiation, the severity of erythema is evaluated and graded (0: no reaction; 1 : mottled erythema; 2 : diffuse erythema; 3 : edematous erythema; and 4: severe necrotic erythema). ${ }^{29}$

The test also carried out using healthy, adult, male or female Sprague Dawley rats. The fur on the backs and abdomens of the rat is removed. After drug administration, the rats are irradiated under anesthesia. Later, the skin reactions are observed at 2, 24, 48 , and 72 hours after the end of the light irradiation, according to the Draize method (Table 3). ${ }^{30}$

\section{In Vitro Skin Irritation Assay}

The test is based on reconstructed human epidermis (RhE) system, which mimics the physiological and biochemical properties of the upper parts of the human skin. This system uses human-derived non-transformed keratinocytes as a cell source to reconstruct an epidermal model. ${ }^{31}$ Normal human epidermal keratinocytes (NHEK) are grown in a fresh medium of culture and maintained in a humidified atmosphere with $7.5 \% \mathrm{CO}_{2}$ at $37^{\circ} \mathrm{C}$; passages $1-3$ are used for the experiments. ${ }^{32}$ On the day of the experiment, $100 \mu \mathrm{L}$

Table 3: Evaluation of skin reactions according to the Draize's criteria

\begin{tabular}{lllll}
\hline \multicolumn{2}{c}{ Score for erythema formation } & & \multicolumn{2}{c}{ Score for edema formation } \\
\cline { 1 - 2 } \cline { 5 - 5 } Score & Skin reactions & & Score & Skin reactions \\
\hline 0 & No erythema & & 0 & No edema \\
1 & Very slight erythema & & 1 & Very slight edema \\
2 & Well-defined erythema & 2 & Slight edema \\
3 & Moderate to severe & 3 & Moderate edema \\
& $\begin{array}{l}\text { erythema } \\
4\end{array}$ & & & \\
& Severe erythema to & 4 & Severe edema \\
\hline
\end{tabular}

of the test compound is applied to the skin models and allowed to stand for 35 minutes at room temperature. Later, the skin models are washed eight times with $600 \mu \mathrm{L}$ PBS each and additionally immersed five times into $60 \mathrm{~mL}$ fresh PBS. After a postexposure incubation of 42 hours at $37^{\circ} \mathrm{C}$ and $5 \% \mathrm{CO}_{2}$, tissue viability is evaluated. ${ }^{33}$ After the exposure, cell viability is determined by the 3-(4,5-dimethylthiazol-2-yl)-2,5-diphenyltetrazolium bromide (MTT) assay. ${ }^{32}$

\section{In Vitro Skin Corrosion Test}

This test is performed according to the OECDTG 431. ${ }^{34}$ Reconstructed human epidermis is obtained from an in vitro process in which keratinocytes are cultured on an inert polycarbonate medium. ${ }^{35}$

Reconstructed human epidermis (RhE) can be obtained from commercial sources. Commercially available RhE are transferred to wells of 6-well culture plates containing culture media and preincubated in a $5 \% \mathrm{CO}_{2}$ incubator overnight according to the manufacturer's protocol. After preincubation, the RhE inserts are exposed to test compound for 3 minutes, and separately for 60 minutes. Three skin replicates are used for each of the test compound and control groups. After exposure, test compounds are removed by repeated rinsing with PBS, and skin models are transferred to the new plates for the viability test. MTT assay is used for quantifying tissue viability. ${ }^{36,37}$

The test compound is considered to be corrosive to the skin if the viability is $<50 \%$ after 3 minutes of exposure. Although the viability after 3 minutes of exposure is $>50 \%$, it is corrosive if the viability is $<15 \%$ after 60 minutes of exposure. It will be non-corrosive if the viability is $>50 \%$ after 3 minutes of exposure and $>15 \%$ after 60 minutes of exposure. In the skin irritation test, the test compound is considered to be irritant to the skin if the tissue viability after exposure/postincubation is $\leq 50 \%$. The test compound is non-irritant if the viability is $>50 \%$ in accordance with OECD TG $439 .^{37}$

Caution: This model should be free of microbial contamination.

\section{In Vitro Skin Sensitization Test}

According to the OECD TG $442 \mathrm{C}$ [direct peptide reactivity assay (DPRA)], 442D (ARE-Nrf2 luciferase test method), and 442E (in vitro skin sensitization assays), in vitro skin sensitization test is performed. ${ }^{38-40}$ This model is used to identify the adverse outcome pathway (AOP) with established key events (KEs) aimed at enhancing data understanding and interpretation mechanisms and helping to develop reliable tests.

\section{Direct Peptide Reactivity Assay}

This method is based on chemical reaction and not required cell lines. The results are based on the reactivity of the test compound with either cysteine or lysine following 24 hours of incubation at $22.5-30^{\circ} \mathrm{C}$. The free peptide concentration is measured by highperformance liquid chromatography (HPLC). ${ }^{40,41}$

\section{ARE-Nrf2 Luciferase Test Method}

This method initiates keratinocyte induction of cytoprotective gene pathways linked to skin sensitization. $\mathrm{Cl} 27$ cells (Nrf2/ luciferase reporter model) are seeded in 96-well plates of different concentrations of a test compound or dimethyl sulfoxide (DMSO), as a negative control. The luciferase cell culture lysis reagent is used as a blank. After $24 / 48$ or 72 hours of the treatment, the medium is removed and washed with $100 \mu \mathrm{L}$ of cold PBS. Later, cells are lysed with luciferase cell culture lysis at room 
temperature. One hundred microliters of luciferase assay reagent are added into each well for a sufficient time (e.g., 20 minutes at room temperature) and luciferase activity is determined. Luciferase bioluminescence measurements are performed at room temperature using a luminometer. Activity is expressed as relative light units (RLU) emitted from total assays and it is calculated against background activity. Or the measurement is based on manufacturer's instruction. ${ }^{39,42,43}$

\section{In Vitro Skin Sensitization Assays}

THP-1, a human monocytic leukemia cell line, is used for the experiment [also known as human cell line activation test ( $h-C L A T)$ ]. THP-1 cells are cultured in RPMI 1640 and supplemented with heat-inactivated fetal bovine serum, 2-mercaptoethanol, and antibiotic-antimycotic. The test compound is dissolved in DMSO or saline. THP- 1 cells $\left(1 \times 10^{6}\right.$ cells $/ \mathrm{mL} /$ well $)$ are treated with a 2 -fold serial dilution of different concentrations of a test compound in 24-well plates and incubated for 24 hours at $37^{\circ} \mathrm{C}$ under $5 \% \mathrm{CO}_{2}$. The cells are washed with staining buffer, PBS containing $0.1 \%$ bovine serum albumin, stained with propidium iodide, and cell viability is analyzed by flow cytometry. . $^{3,44}$

\section{In Vitro Phototoxicity}

According to OECD TG 432, phototoxicity is a toxic response elicited by systemically or topically administered photoreactive substances after the exposure of the body to environmental light. ${ }^{45}$

\section{In Vitro 3 T3 Neutral Red Uptake (NRU) Phototoxicity Test}

Balb/3T3 clone A31 cells are used for the experiment. The cells in the logarithmic growth phase are seeded in a 96-well assay plate at $10^{4}$ cells/well and cultured in Dulbecco's modified Eagle's medium (DMEM) for 24 hours at $37^{\circ} \mathrm{C}$ and $5 \% \mathrm{CO}_{2}$ for the formation of monolayers. Then, wells are washed with PBS before adding the test compounds.

Test compound is prepared freshly prior to the experiment. The test compound is dissolved in PBS or DMSO, and then diluted in PBS to $1 \% \mathrm{v} / \mathrm{v}$. For each test compound, one culture plate is prepared for UV irradiation and one for non-UV irradiation, and the highest concentration of each plate is determined from the cytotoxicity of each test compound.

Aspirate the routine culture medium from the plates and added $100 \mu \mathrm{L}$ of each concentration of test compounds according to a predefined plate layout and incubated for 60 minutes before UV irradiation/non-UV irradiation. One plate is irradiated with ultraviolet A (UVA) $1.7 \mathrm{~mW} / \mathrm{cm}^{2}$ for 50 minutes and other plate is maintained in dark at room temperature without irritation. Later, the plates are washed with PBS and the fresh medium is added to each well before overnight incubation. After 24 hours, the cells are washed with PBS, neutral red is added, and cells are incubated for 3 hours. Cytotoxicity is measured according to neutral red absorption at $540 \mathrm{~nm}$ using a microplate reader. Cytotoxicity in this test is expressed as a concentration-dependent reduction of the uptake of the vital dye "neutral red" when measured 24 hours after treatment with the test compound and irradiation. ${ }^{45-47}$

\section{Photoallergy}

Photoallergy is uncommon. Evaluation of photoallergy potential for cosmetic ingredients is recommended by the Personal Care Products Council (PCPC) use of the reactive oxygen species (ROS) assay. $^{48}$
The ROS assay is intended to detect both singlet oxygen $\left({ }^{1} \mathrm{O}_{2}\right)$ and superoxide $\left(\mathrm{O}_{2}^{-}\right)$generated from photo-irradiated chemicals. Briefly, ${ }^{1} \mathrm{O}_{2}$ is measured in an aqueous solution by spectrophotometrically monitoring the bleaching of p-nitrosodimethylaniline at 440 $\mathrm{nm}$ using imidazole as a selective acceptor of ${ }^{1} \mathrm{O}_{2}$. Different concentration of test compound $(2-200 \mathrm{mM})$ is mixed with p-nitrosodimethylaniline $(50 \mathrm{mM})$ and imidazole $(50 \mathrm{mM})$ in $20 \mathrm{mM}$ sodium phosphate buffer ( $\mathrm{pH}$ 7.4). Two hundred microliters of the sample are transferred in a well of 96-well plate and absorbance is measured at $440 \mathrm{~nm}$ using a microplate reader. The plate irradiated with simulated sunlight for 1 hour and absorbance is measured at $440 \mathrm{~nm}^{49,50}$

For the determination of $\mathrm{O}_{2}^{-}$, samples containing the tested compound (2-200 mM), nitroblue tetrazolium (NBT) and, sodium phosphate buffer is irradiated with the simulated sunlight for 1 hour and the reduction in NBT is measured by the increase in absorbance at $560 \mathrm{~nm}$ in the same manner as the ${ }^{1} \mathrm{O}_{2}$ determination. ${ }^{50,51}$

ROS generation of ${ }^{1} \mathrm{O}_{2}$ (Decrease of $A_{440} \times 1000=\left[A_{440(-)}-\right.$ $\left.A_{440(+)}-(a-b)\right] \times 1000$, where $A_{440(-)}$ : absorbance before light exposure at $440 \mathrm{~nm} ; A_{440(+)}$ : absorbance after light exposure at $440 \mathrm{~nm}$; $a$ : blank before light exposure; and $b$ : Blank after exposure) and $\mathrm{O}_{2}^{-}$anion (increase of $A_{560} \times 1,000=\left[A_{560(+)}-A_{560(-)}-(b-a)\right] \times$ 1,000 , where $A_{560(-)}$ : absorbance before light exposure at $560 \mathrm{~nm}$, $A_{560(+)}$ : absorbance after light exposure at $560 \mathrm{~nm}, a$ : blank before light exposure; and $b$ : blank after exposure) is calculated mathematically. ${ }^{48}$

\section{Skin Genotoxicity}

Genotoxicity refers to processes that alter the structure, information content, or segregation of DNA and that are not necessarily linked with mutagenicity. ${ }^{52}$ Genotoxicity can be performed in yeast, bacterial, and mammalian cells. The bacterial reverse mutation test (Ames test), the Escherichia coli reverse mutation assay, the in vitro mammalian chromosome aberration test, and the in vitro mammalian cell gene mutation test are used to study the genotoxicity. ${ }^{53}$ Genotoxins can be classified into three groups on the basis of their effects. They are mutagens or mutation-causing agents, carcinogens or cancer-causing agents, and teratogens or birth defect-causing agents. ${ }^{54}$

Genotoxicity testing is an important building block for ensuring the protection of cosmetic products while preventing damage to DNA and its possible undesired health effects, such as genetic defects and cancer. Provided that the EU Cosmetics Directive under no circumstances permits the animal testing for cosmetics, under in vivo testing for cosmetic ingredients, including confirmatory genotoxicity tests, is no longer feasible. ${ }^{55}$

\section{Animal Welfare Considerations}

Use of animals for dermal (skin) toxicity test may cause potential pain and damage to the skin that result from cutaneously applied materials/dermal formulations. Hence, careful monitoring is mandated by animal welfare regulations. To prevent animals from suffering, many of the regulatory agencies suggest performing the toxicity testing using alternative models which meet the goals (the 3 R's, i.e., reduction, refinement, replacement) of alternatives to animal testing. ${ }^{56}$ To minimize the use of animals in research and to implement alternatives, in 2012 and 2016 United States Environmental Protection Agency published a guideline for waving acute dermal toxicity tests titled "Guidance for waiving or bridging of mammalian acute toxicity tests for pesticides and pesticide 
products" and "Guidance for waiving acute dermal toxicity tests for pesticide formulations and supporting retrospective analysis", respectively. ${ }^{57}$ This indicates that, replacement is possible in an animal experiment if a suitable alternative is available to perform the toxicity testing by alternative methods.

\section{Conclusion}

Toxicity testing of an investigational new drug/a formulation is essential for the drug development process. The data on toxicity are very essential to observe the physiological/biochemical/ pathological/toxicological effect of any test compound and the study data are helpful to understand the toxicokinetic properties of test compounds and predict the human safe dose. In this article, the method used to study the safety and toxicological properties of dermal formulation(s) are summarized.

\section{References}

1. Riebeling C, Luch A, Tralau T. Skin toxicology and 3Rs-current challenges for public health protection. Exp Dermatol 2018;27(5): 526-536. DOI: 10.1111/exd.13536.

2. Paudel KS, Milewski M, Swadley CL, Brogden NK, Ghosh P, Stinchcomb AL. Challenges and opportunities in dermal/transdermal delivery. Ther Deliv 2010;1(1):109-131. DOI: 10.4155/tde.10.16.

3. Arunachalam A, Karthikeyan M, Kumar DV, Prathap M, Sethuraman S, Ashutoshkumar S, et al. Transdermal drug delivery system: a review. Cur Pharma Res 2010;1(1):70. DOI: 10.33786/JCPR.2010.v01i01.015.

4. List of Pharmaceutical dosage forms. Available in https://view. officeapps.live.com/op/view.aspx?src=https $\% 3 A \% 2 F \% 2 F w w w . e m a$. europa.eu\%2Fen\%2Fdocuments\%2Fother\%2Flist-pharmaceuticaldosage-forms_en.xls. Last assessed on 24-05-2020.

5. Sachan R, Bajpai M. Transdermal drug delivery system: a review. IJRDPL 2013;3:748-765.

6. Dermal Fillers Market Size, Growth \& Revenue Analysis 2026. https:// www.fortunebusinessinsights.com/industry-reports/dermal-fillersmarket-100939. Last assessed on 24-05-2020.

7. Dermal Fillers Market Value to Hit US $\$ 10.4 \mathrm{~B}$ by 2026 : Global Market Insights, Inc. Available in https://www.prnewswire.com/ news-releases/dermal-fillers-market-value-to-hit-us-10-4b-by2026-global-market-insights-inc-300999555.html. Last assessed on 24-05-2020.

8. Bilal M, Iqbal HM. An insight into toxicity and human-health-related adverse consequences of cosmeceuticals-a review. Sci Total Environ 2019;670:555-568. DOI: 10.1016/j.scitotenv.2019.03.261.

9. Lohani A, Verma A, Joshi H, Yadav N, Karki N. Nanotechnologybased cosmeceuticals. ISRN Dermatol 2014;2014:843687. DOI: 10.1155/2014/843687.

10. Parasuraman S. Toxicological screening. J Pharmacol Pharmacother 2011;2(2):74-79. DOI: 10.4103/0976-500X.81895.

11. Acute Dermal Toxicity. Available in https://www.schc.org/assets/ docs/ghs_info_sheets/Acute\%20Dermal\%20Toxicity\%20(Final\%20 2018-03).pdf. Last assessed on 25-05-2020.

12. Wakure BS, Bhatia NM. Acute dermal toxicity and irritability studies of $\mathrm{Ag}_{2} \mathrm{Ga}$ nanoneedle mediated silver formulation as per OECD 402 and 404 protocols. Int J Pharm Sci Res 2018;9(9):4015-4020.

13. OECD Test No. 402: Acute Dermal Toxicity, OECD Guidelines for the Testing of Chemicals, Section 4. Paris: OECD Publishing. DOI: 10.1787/9789264070585-en.

14. GHS Classification Criteria for Acute Toxicity. Available in https:// www.chemsafetypro.com/Topics/GHS/GHS_classification_criteria_ acute_toxicity_category.html. Last assessed on 25-05-2020; Acute Dermal Toxicity. Available in https://www.schc.org/assets/docs/ ghs_info_sheets/Acute\%20Dermal\%20Toxicity\%20(Final\%20201803).pdf. Last assessed on 25-05-2020.
15. Combes RD. Alternatives to Animal Testing. In Comprehensive Medicinal Chemistry II Taylor JB, Triggle DJ, ed. . Elsevier; 2007. p. 473. ISBN: 978-0-08-045044-5.

16. OECD Test No. 411: Subchronic Dermal Toxicity: 90 -day Study, OECD Guidelines for the Testing of Chemicals, Section 4. OECD Publishing, Paris 1981. DOI: https://doi.org/10.1787/9789264070769-en.

17. Health Effects Test Guidelines: OPPTS 870.3250 90-Day Dermal Toxicity [EPA 712-C-98-202]. Available in https://www.regulations. gov/contentStreamer?documentld=EPA-HQ-OPPT-2009-01560013\&contentType $=$ pdf. Last assessed on 25-05-2020.

18. Deshpande PO, Mohan V, Thakurdesai P. Preclinical safety assessment of standardized extract of Centella asiatica (L.) urban leaves. Toxicol Int 2015;22(1):10-20. DOI: 10.4103/0971-6580.172251.

19. Zuang V, Alonso M, Botham PA, Eskes C, Fentem J, Liebsch M, et al., Skin Irritation/Corrosion. Skin Irritation/Corrosion - European Commission. Available in ec.europa.eu. Last assessed on 25-05-2020.

20. OECD Test No. 404: Acute Dermal Irritation/Corrosion, OECD Guidelines for the Testing of Chemicals. 2002, Available in https:// ntp.niehs.nih.gov/iccvam/suppdocs/feddocs/oecd/oecdtg404.pdf. Last assessed on 25-05-2020.

21. Wang J, Li Z, Sun F, Tang S, Zhang S, Lv P, et al. Evaluation of dermal irritation and skin sensitization due to vitacoxib. Toxicol Rep 2017;4:287-290. DOI: 10.1016/j.toxrep.2017.06.003.

22. Globally Harmonized System of Classification and Labelling of Chemicals (GHS) (Rev.5) (2013). Available in http://www.unece.org/ trans/danger/publi/ghs/ghs_rev05/05files_e.html. Last assessed on 26-05-2020.

23. OECD Test No. 442B: Skin Sensitization: Local Lymph Node Assay: BrdU-ELISA or -FCM, OECD Guidelines for the Testing of Chemicals, Section 4. Paris: OECD Publishing; 2018. DOI: https://doi. org/10.1787/9789264090996-en.

24. OECD (1092) Test No. 406. Skin Sensitisation. OECD Guidelines for the Testing of Chemicals. Available in https://ntp.niehs.nih.gov/ iccvam/suppdocs/feddocs/oecd/oecdtg406.pdf. Last assessed on 26-05-2020.

25. ICCVAM-Recommended Test Method ProtocolUpdated Protocol for the Murine Local Lymph Node Assay. Available in https://ntp.niehs. nih.gov/iccvam/docs/protocols/lnaupdated.pdf. Last assessed on 27-05-2020.

26. Kim K, Park H, Lim KM. Phototoxicity: its mechanism and animal alternative test methods. [published correction appears in Toxicol Res. 2015;31(3):321] Toxicol Res 2015;31(2):97-104. DOI: 10.5487/ TR.2015.31.2.097.

27. Rittié L, Fisher GJ. Natural and sun-induced aging of human skin. Cold Spring Harb Perspect Med 2015;5(1):a015370. DOI: 10.1101/ cshperspect.a015370.

28. Kornhauser A, Wamer WG, Giles AL. Psoralen Phototoxicity: correlation with serum and epidermal 8-methoxypsoralen and 5-methoxypsoralen in the guinea pig. Science 1982;217(4561): 733-735. DOI: 10.1126/science.7100920.

29. Horio T, Miyauchi H, Asada Y, Aoki Y, Harada M. Phototoxicity and photoallergenicity of quinolones in guinea pigs. J Dermatol Sci 1994;7(2):130-135. DOI: 10.1016/0923-1811(94)90086-8.

30. Yonezawa $\mathrm{Y}$, Katou $\mathrm{H}$, Kuga K. Multi-site study of an in vivo phototoxicity evaluation in Sprague-Dawley (SD) rats aimed at incorporating the phototoxicity assessments: effects of repeated administration and toxicokinetic blood collection on drug-induced phototoxicity. Fundam Toxicol Sci 2019;6(6):197-206. DOI: 10.2131/ fts.6.197.

31. OECD Test No. 439: In Vitro Skin Irritation: Reconstructed Human Epidermis Test Method, OECD Guidelines for the Testing of Chemicals, Section 4. Paris: OECD Publishing; 2019. DOI: https://doi. org/10.1787/9789264242845-en.

32. do Nascimento Pedrosa T, Catarino CM, Pennacchi PC, de Assis $\mathrm{SR}$, Gimenes F, Consolaro $\mathrm{ME}$, et al. A new reconstructed human epidermis for in vitro skin irritation testing. Toxicol In Vitro 2017;42: 31-37. DOI: 10.1016/j.tiv.2017.03.010. 
33. Schmidt FF, Nowakowski S, Kluger PJ. Improvement of a three-layered in vitro skin model for topical application of irritating substances. Front Bioeng Biotechnol 2020;8:388. DOI: 10.3389/fbioe.2020.00388.

34. OECD Test No. 431: In Vitro Skin Corrosion: Human Skin Model Test. OECD Publishing, Paris 2004. DOI: https://doi. org/10.1787/9789264071148-en.

35. Reconstructed human epidermis: An efficient prediction tool. Life Sci 2011;40(6):1-2.

36. Kim H, Choi J, Lee H, Park J, Yoon B, Jin SM, et al. Skin corrosion and irritation test of nanoparticles using reconstructed three-dimensional

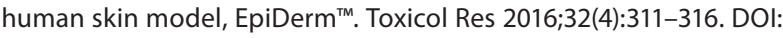
10.5487/TR.2016.32.4.311.

37. Choi J, Kim H, Choi J, Oh SM, Park J, Park K. Skin corrosion and irritation test of sunscreen nanoparticles using reconstructed 3D human skin model. Environ Health Toxicol 2014;29:e2014004. DOI: 10.5620/ eht.2014.29.e2014004.

38. OECD, Test No. 442E: In Vitro Skin Sensitisation: In Vitro Skin Sensitisation assays addressing the Key Event on activation of dendritic cells on the Adverse Outcome Pathway for Skin Sensitisation, OECD Guidelines for the Testing of Chemicals, Section 4. Paris: OECD Publishing; 2018. DOI: 10.1787/9789264264359-en.

39. OECD, Test No. 442D: In Vitro Skin Sensitisation: ARE-Nrf2 Luciferase Test Method, OECD Guidelines for the Testing of Chemicals, Section 4. Paris: OECD Publishing; 2018. DOI: https://doi. org/10.1787/9789264229822-en.

40. OECD, Test No. 442C: In Chemico Skin Sensitisation: Assays addressing the Adverse Outcome Pathway key event on covalent binding to proteins, OECD Guidelines for the Testing of Chemicals, Section 4. Paris: OECD Publishing; 2019. DOI: https://doi. org/10.1787/9789264229709-en.

41. Bauch C, Kolle SN, Ramirez T, Eltze T, Fabian E, Mehling A, et al. Putting the parts together: combining in vitro methods to test for skin sensitizing potentials. Regul Toxicol Pharmacol 2012;63(3):489-504. DOI: 10.1016/j.yrtph.2012.05.013.

42. Motahari P, Sadeghizadeh M, Behmanesh M, Sabri S, Zolghadr F. Generation of stable ARE- driven reporter system for monitoring oxidative stress. Daru 2015;23(1):38. DOI: 10.1186/s40199-0150122-9.

43. Zagoura D, Canovas-Jorda D, Pistollato F, Bremer-Hoffmann S, Bal-Price A. Evaluation of the rotenone-induced activation of the Nrf2 pathway in a neuronal model derived from human induced pluripotent stem cells. Neurochem Int 2017;106:62-73. DOI: 10.1016/j. neuint.2016.09.004.

44. Takenouchi O, Miyazawa M, Saito K, Ashikaga T, Sakaguchi H. Predictive performance of the human cell line activation test (h-CLAT) for lipophilic chemicals with high octanol-water partition coefficients. J Toxicol Sci 2013;38(4):599-609. DOI: 10.2131/jts.38.599.
45. OECD, Test No. 432: In Vitro $3 T 3$ NRU Phototoxicity Test, OECD Guidelines for the Testing of Chemicals, Section 4. Paris: OECD Publishing; 2019. DOI: https://doi.org/10.1787/9789264071162-en.

46. Toyoda A, Sugiyama M, Furihata S, Nishizumi K, Omori T, Itagaki H. Development of a modified 3T3 neutral red uptake phototoxicity test protocol for evaluation of poorly water-soluble substances. J Toxicol Sci 2017;42(5):569-577. DOI: 10.2131/jts.42.569.

47. Ates $G$, Vanhaecke $T$, Rogiers V, Rodrigues RM. Assaying cellular viability using the neutral red uptake assay. In Cell Viability Assays. New York, NY: Humana Press; 2017. pp. 19-26.

48. ROS (Reactive Oxygen Species) assay for photosafety in OECD guideline for the testing of chemicals. Available in http://www. oecd.org/env/ehs/testing/Draft\%20TG\%20ROS\%20161012.pdf. Last assessed on 27-05-2020.

49. Onoue S, Suzuki G, Kato M, Hirota M, Nishida $H$, Kitagaki $M$, et al. Non-animal photosafety assessment approaches for cosmetics based on the photochemical and photobiochemical properties. Toxicol In Vitro 2013;27(8):2316-2324. DOI: 10.1016/j.tiv.2013.10.003.

50. Onoue S, Hosoi K, Wakuri S, Iwase Y, Yamamoto T, Matsuoka N, et al. Establishment and intra-/inter-laboratory validation of a standard protocol of reactive oxygen species assay for chemical photosafety evaluation. J Appl Toxicol 2013;33(11):1241-1250. DOI: 10.1002/ jat.2776.

51. Lee YS, Yi JS, Lim HR, Kim TS, Ahn IY, Ko K, et al. Phototoxicity evaluation of pharmaceutical substances with a reactive oxygen species assay using ultraviolet A. Toxicol Res 2017;33(1):43-48. DOI: 10.5487/TR.2017.33.1.043.

52. Pellevoisin C, Bouez C, Cotovio J. Cosmetic industry requirements regarding skin models for cosmetic testing. In Skin Tissue Models. Academic Press; 2018. pp. 3-37.

53. Genotoxicity. Available in http://alttox.org/mapp/toxicity-endpointstests/genotoxicity/. Last assessed on 27-05-2020.

54. Barabadi $H$, Najafi M, Samadian H, Azarnezhad A, Vahidi $H$, Mahjoub MA, et al. A systematic review of the genotoxicity and antigenotoxicity of biologically synthesized metallic nanomaterials: are green nanoparticles safe enough for clinical marketing? Medicina (Kaunas) 2019;55(8):439. DOI: 10.3390/medicina55080439.

55. Chave J. Advances in genotoxicity testing: a keystone of our work on alternatives. Cosmet Eur Res Newslet 2017(2):Available in https://www.cosmeticseurope.eu/files/9314/9675/8761/Research_ Newsletter_issue_2.pdf. Last assessed on 27-05-2020.

56. Auletta CS. Current in vivo assays for cutaneous toxicity: local and systemic toxicity testing. Basic Clin Pharmacol Toxicol 2004;95(5): 201-208. DOI: 10.1111/j.1742-7843.2004.pto950501.x.

57. Bridging or waiving data requirements. Available in https://www.epa. gov/pesticide-registration/bridging-or-waiving-data-requirements. Last assessed on 27-05-2020. 